\title{
Cascaded Segmentation Network BASED ON DOUBLE BRANCH BOUNDARY ENHANCEMENT
}

\author{
Li Zeng ${ }^{1}$, Hongqiu Wang ${ }^{1}$, Xin Wang ${ }^{3}$, Miao Tian ${ }^{1 *}$ and Shaozhi $\mathrm{Wu}^{1,2^{*}}$ \\ ${ }^{1}$ University of Electronic Science and Technology of China, \\ Chengdu, 611731, China \\ ${ }^{2}$ Yangtze Delta Region Institute (Quzhou), University of Electronic Science and \\ Technology of China, Quzhou, Zhejiang 324000, China \\ ${ }^{3}$ Department of Abdminal Oncology, Cancer Center, \\ West China Hospital, Sichuan University, China
}

\begin{abstract}
Cervical cancer is one of the most common causes of cancer death in women. During the treatment of cervical cancer, it is necessary to make a radiation plan based on the clinical target volume (CTV) on the CT image. At present, CTV is manually sketched by physicists, which is time-consuming and laborious. With the help of deep learning model, computer can accurately draw the outline of CTV in Colleges and universities. The CDBNet proposed in this paper is a cascaded segmentation network based on double-branch boundary enhancement. First, classification network determines whether a single image contains a region of interest (ROI), and then the segmentation network uses DBNet to segment more accurately at the ROI contour. In this paper, we propose CDBNet, a cascaded segmentation network based on doublebranch boundary enhancement. First, classification network determines whether a single image contains a region of interest $(R O I)$, and then the segmentation network uses DBNet to segment more accurately at the ROI contour. The CDBNet proposed in this paper was verified on the cervical cancer dataset provided by the Department of Radiation Oncology, West China Hospital, Sichuan Province. The average dice and 95HD of the delineation results are $86.12 \%$ and $2.51 \mathrm{~mm}$. At the same time, the classification accuracy rate of whether the image contains ROI can reach 93.19\%, and the average Dice of the image containing ROI can reach $70 \%$.
\end{abstract}

\section{KEYWORDS}

CTV delineation, cascade, segmentation, boundary enhancement.

\section{INTRODUCTION}

The International Agency for Research on Cancer (IARC) estimates that by 2020, more than 600,000 women worldwide have been diagnosed with cervical cancer, and about 340,000 women have died from the disease. Cervical cancer is one of the most common causes of cancer death in women. Early detection and treatment of the diseased can greatly improve the survival rate of patients. Cervical cancer can be prevented by the HPV vaccine, but less than $25 \%$ of girls in the world can get HPV vaccine. In the diagnosis and treatment of cervical cancer, Computed Tomography (CT) is one of the most widely used imaging methods and plays an important role in assisting diagnosis [1]. To facilitate the assessment of cancer and the development of treatment plans, it is necessary to accurately locate the lesion area in the CT image [2]. Currently, medical physicists mainly rely on the manual delineation of CT images to determine the lesion area. The David C. Wyld et al. (Eds): COMIT, CRBL, BIOM, WiMNeT, SIP, AISO, NLDM - 2021 pp. 249-261, 2021. CS \& IT - CSCP 2021 
lesion area is also called a region of interest (ROI) in the segmentation task. The closer to the real ROI delineation, the more accurately the lesion can be treated and the better the surrounding healthy tissue can be protected. However, this manual delineation method is highly subjective and labor-intensive, which affects the efficiency of diagnosis. Therefore, a standardized automatic segmentation method is very necessary.

In recent decades, with the development of Computer-Aided Diagnosis (CAD) [3], some automated segmentation methods based on machine learning have been used in ROI segmentation tasks, including traditional machine learning methods and deep learning methods. Hong [4] used fuzzy C-means clustering, and Bilello et al. [5] used intensity-based histograms and lesion contour refinement to segment. Due to the simple structure of traditional machine learning algorithms, it can only extract some simple features, such as texture, contour and other features, resulting in limited segmentation effects, and there is still a lot of room for improvement.

With the development of deep learning, many ROI segmentation methods based on Convolutional Neural Networks (CNN) have been proposed. Jonathan Long et al. [6] proposed a Full Convolutional Network (FCN) to supervise the training of the model at the pixel level. In the same year as FCN, Olaf Ronneberger et al. proposed UNet [7], which uses skip connection to combine feature maps in the encoding and decoding paths. Semantic information such as positions and contours that are ignored in the encoding path are compensated in the decoding path. Unet performs very well in the field of medical image segmentation, but many researchers believe that Unet and FCN can be improved. Dolz, Jose et al. combined UNet and DenseNet architecture [8] to get DenseUNet [9]. The Dense block in DenseNet can retain more semantic information in the encoding path. Zongwei Zhou et al. proposed the UNet++ [10] network, which uses more nodes to replace jump connections in Unet, so that more semantic information of each feature map can be retained in the network. These methods of retaining semantic information also retain a lot of redundant or erroneous noise information. Therefore, there are many ways to improve segmentation accuracy by supplementing semantic information. Meng et al. [11] used the local path and the global path to complement each other to obtain sufficient 3D spatial information, but the training of 3D network requires higher computer hardware. KiUnet proposed by Jose [12] et al. adds a complete convolution branch to supplement the contour information. The complete convolution part uses a feature map with a larger size, which will cause a larger computational cost. In addition, the model mixes the two branches without deep supervision of the complete convolution branch, which will cause a lot of noise in the boundary features extracted by the branch. In addition, KiUnet mixes the two branches without deep supervision of the complete convolution branch, which will cause a lot of noise in the boundary features extracted by the branch.

The segmentation accuracy of the ROI area is not only reflected in the accuracy of the ROI outline, but also in whether all the slices containing the lesion can be found. In clinical data, the cervical cancer dataset is case-based. A case contains multiple CT slices. In addition to accurately delineating the outline of the lesion area on the slices with lesions, it is also necessary to determine which slices have lesions. Many researchers believe that data balancing can solve this problem. Tran et al. [13] resample the data, that is, exclude $2 / 3$ of the no-ROI slices to produce a more balanced dataset. But it is not sure that the ratio is the best one, and more experiments are needed to find out the ratio of ROI slices to no-ROI slices. Wardhana et al. [14] apply the class weight that adjusting the cost of the class error. And similarly [15][16], these methods also require the manual setting of weights. Some people use feature selection to reduce the number of no-ROI slices. Chlebus [17] trained a conventional random forest classifier (RF) with 256 trees using 36 hand-crafted features to filter false positive. However, hand-crafted features may not be available for other datasets. 
So far, there are few studies on deep learning models for cervical cancer target segmentation. In 2019, Rhee, DJ et al. [18] integrated the CNN network into an auto-contouring tool. In 2020, Peking Union Medical College Hospital [19] was inspired by Unet and double-path network DPN on the basis of CNN and proposed a network DpnUNet designed to perform advanced semantic feature extraction and high-quality CTV delineation.

We propose a novel cascade segmentation network (CDBNet, Cascade Double-Branch Net) based on double-branch boundary enhancement to perform cervical cancer clinical target volume (CTV) segmentation. We use a cascade structure to complete the segmentation of the ROI. The cascade structure contains a classification module and a segmentation module. The classification module can predict whether each CT slice contains ROI, so that the segmentation module can focus on the ROI segmentation. In addition, we propose a novel double-branch boundary enhancement module, which focuses on segmenting the boundary part of the ROI to improve the segmentation accuracy. The main contributions of this paper can be summarized into the following two points:

1) We designed a cascade structure to determine whether the CT slice contains ROI. Among them, we use the segmentation network + post-processing method to perform the classification task, which improves the classification accuracy rate and reduces the classification false positive rate.

2) We propose a novel double-branch boundary enhancement segmentation network (DBNet). The boundary branch conducts independent training for ROI contour segmentation and effectively integrates with the ROI segmentation network of the main baseline branch. This improves the accuracy of ROI segmentation in CT slices. And we put DBNet into the cascade structure to form a stronger CDBNet.

The rest of the paper is arranged as follows: Section 2 describes the proposed method, Section 3 is related to experimental results, and Section 4 summarizes and discusses.

\section{METHOD}

In this section, we introduce the details of the proposed cascaded double-branch segmentation network (CDB-Net). The network architecture is shown in Figure 1. After the CT slice is input, it is divided into two types with ROI and no-ROI by LCM. LCM is the key module to reduce false positives in classification. Then use DBSM to perform accurate target segmentation on the CT slices of the ROI. 


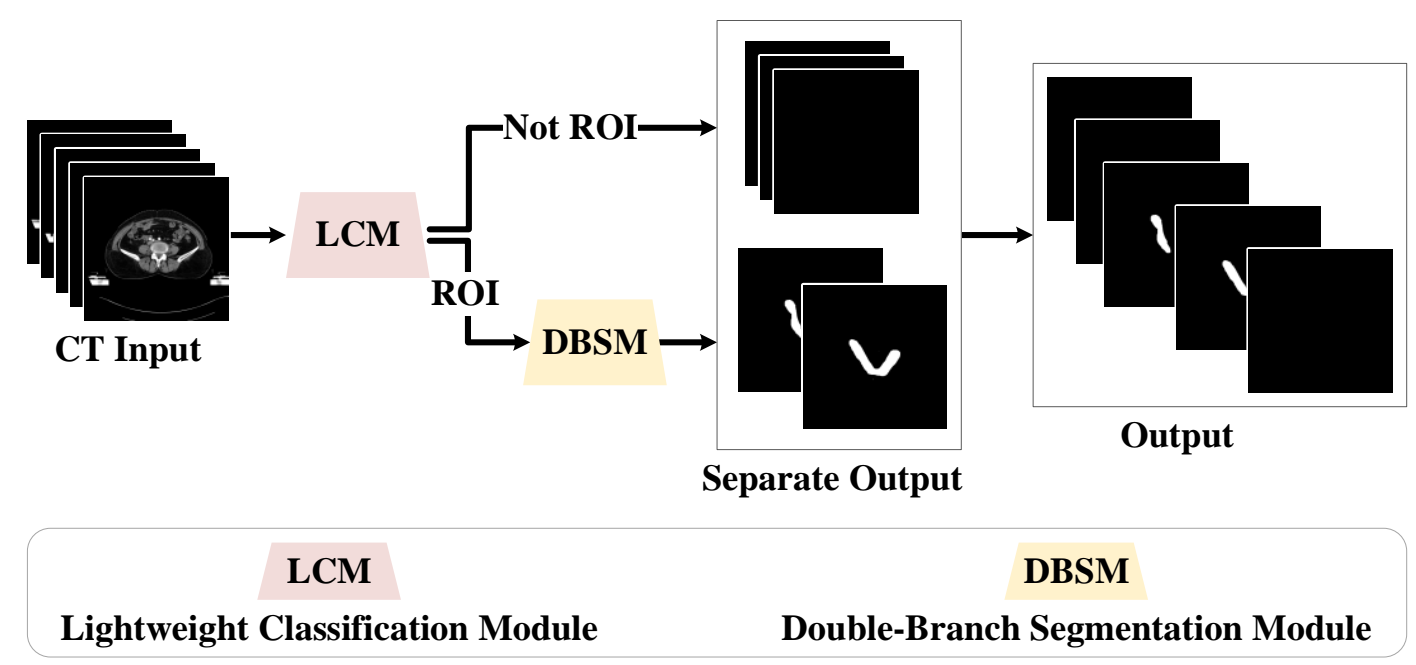

Figure 1 Illustration of Cascade Double-Branch Net (CDBNet). Our proposed framework is cascaded by Lightweight Classification Module (LCM) and Double-Branch Segmentation Module (DBSM). The inputs are Two-dimensional CT slices. Then LCM predicts each CT slice contains ROI or not. The slices containing ROI will be sent to the DBSM to obtain more accurate ROI segmentation.

\subsection{Lightweight Classification Module}

The LCM module is used to determine whether the CT slice contains an ROI so that the DBSM only needs to pay attention to the slice with ROI. Due to the blurred and changeable ROI boundary, the classification method at the CT slice level is not sufficient for accurate classification. Therefore, it is necessary to perform classification at the pixel level and then judge at the slice level, which can improve the classification accuracy. In the LCM module, because it does not require precise segmentation results, the Unet [7] structure with fewer parameters is used to segment CT images. The Unet model consists of a down-sampling encoder and an upsampling decoder, as shown in Figure 2. The Unet down-sampling path extracts and filters features, while the up-sampling path restores size and supplementary features. The InConv operation in Figure 2 converts the input data to a size of $256 \times 256$, and then expands the channel of the feature map through convolution. The DownConv operation convolves and downsamples the feature map twice. The Skip + UpConv operation includes skipping connection and two convolution operations. For example, in the Skip + UpConv process of Dn5, the Dn5 feature map is first up-sampled to obtain the feature map Up4, then it is merged with the feature map Dn4, and finally, convolution is performed. The OutConv operation makes the model output a channel feature map through convolution.

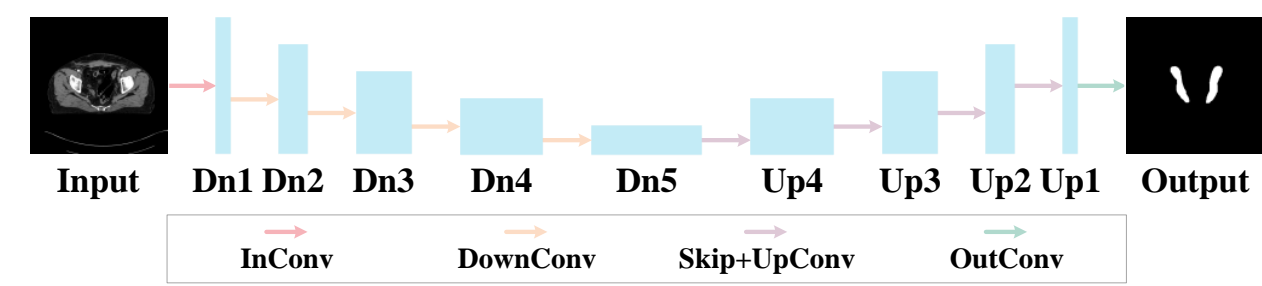

Figure 2. U-net architecture Error! Reference source not found. uses the Encoder-Decoder structure. And it uses skip connections in the decoding path to retain more information. 
Finally, it is judged whether each CT slice contains ROI according to the result of segmentation. Because the area of ROI in a small number of slices is very small, a threshold $n$ is set in this paper to assist classification. The value of $n$ is the optimal threshold determined based on the performance of different thresholds on the validation set. When the sum of ROI in each pixel in a single segmentation prediction image is greater than $\mathrm{n}$, it is considered that the CT slice contains ROI, otherwise, it does not.

\subsection{Double-Branch segmentation Module}

Although the structure of the encoder and decoder has been well done in the field of medical image segmentation, some information will be ignored in the encoding path. The Unet model uses Skip connection to solve this problem. Skip connection supplements the large-size feature map as complete information to the up-sampling decoder path, but we may need to pay more attention to the boundary segmentation in the large-size feature map. Figure 4 vividly shows the correctness of this idea. When the Unet feature map has a smaller size (such as Up3), the target area is not clear; in the Up2 and Up1 stages with a larger feature map, the target area is gradually clear. At this time, the fusion of clearer boundary information will be more beneficial to the training of the network.

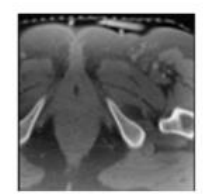

Input

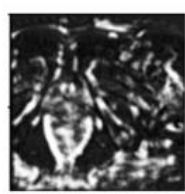

Up3

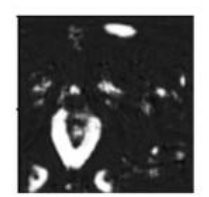

Up2

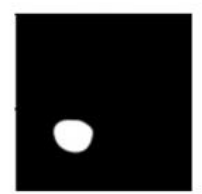

Up1

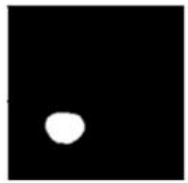

Label

Figure 3. The feature maps of each layer and label. Up3, Up2, Up1 correspond to the feature map in Figure 2

KiUnet mentioned that using a complete convolution branch to provide boundary information can improve segmentation accuracy. But the boundary mentioned in that paper is a feature map with more noise. Therefore, we are inspired by the double-branch structure of KiUnet. Our DBSM includes the baseline branch and border branch. The Baseline branch uses the Unet structure to provide the main ROI segmentation. The Border branch retains the larger layer of the feature map in Unet, and uses the ground truth border image as supervision for training. And merge the baseline branch model with the border branch at each layer to achieve the purpose of effectively enhancing the border. The specific structure diagram is shown in Figure 4. The border branch takes the Input feature map as input, and then performs two down-sampling DownConv operations and two up-sampling Skip+UpConv operations. During down-sampling, the contour information is supplemented in the baseline by ConcatConv-Down operation, and the upsampling is supplemented by ConcatConv-Up. Finally, after the ConcatConv operation, the single-channel feature map is output as the ROI prediction map, and the border branch outputs a contour prediction map. 


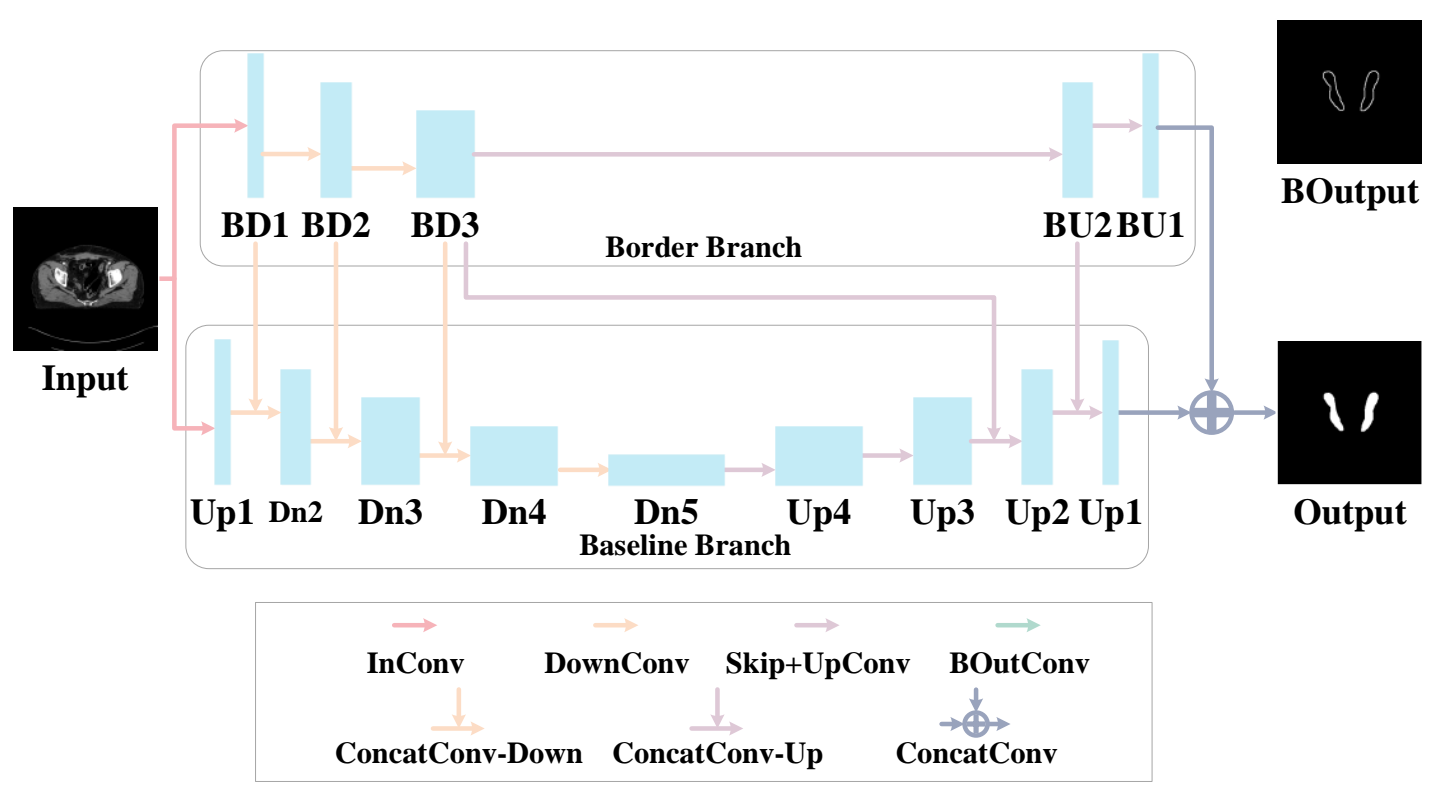

Figure 4. Double-Branch Segmentation Module (DBSM). The DBSM consists of the Baseline branch and the Border branch. The Baseline Branch performs segmentation prediction on the CT slice, and the Border Branch predicts the contour of the ROI. The two branches are merged through ConcatConv-Down, ConcatConv-Up, and ConcatConv. Finally, a more accurate segmentation prediction is output.

\subsection{Loss Function}

The loss function of DBNet training consists of two parts, one is the dice of the predicted contour and the real contour, defined as 1_border, and the other is the dice of the predicted ROI and the real ROI, defined as 1_end. The loss function used in the training process is as in equation (1). Among them, $m$ is the length of the input feature map, $n$ is the width of the input feature map, $c$ is the number of channels, A is the predicted contour map, B is the real contour map marked by the physicist, and 1_border is calculated as equation (2). The calculation of 1_end is similar to equation (2).

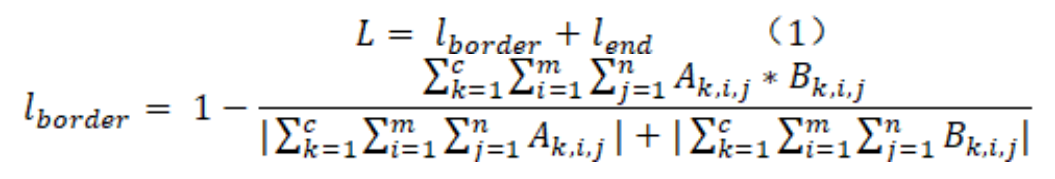

\section{EXPERIMENTS}

The dataset used in this paper comes from the Department of Radiation Oncology, West China Hospital, Sichuan Province. The experimental task is to segment the cervical cancer CTV in this dataset. Section 3.1 describes the dataset and related preprocessing operations. Section 3.2 explains the evaluation indicators used in this article. 3.3 shows the experimental results of cervical cancer clinical target volume segmentation. Section 3.5 shows the results of the ablation experiment.

\subsection{Dataset and preprocessing}

The dataset contains 276 patients, and the data comes from the contouring results of multiple physicists, and each case is contoured by only one physicist. In order to make the model 
universal, the data will only be used when a physicist outlines more than 30 cases, and the final dataset contains 196 patients. After random scrambling, the data of 19 patients were used as the test set, the data of 177 patients were used as the training set and the validation set, and the fivefold cross-validation was used for training. The data is read in from the Dicom medical format. The original size of the CT slice image is $512 \times 512$. Cut the HU range of the CT slice to [$128,256]$, set the HU value less than -128 to -128 , and set it to 256 if the HU value is more than 256. And then normalized to [0, 1]. This paper uses GeForce RTX 2080Ti for network training, the epoch is set to 25 , and batch size is set to the maximum value available for each model.

\subsection{Evaluation}

The values involved in the experiment are all averages in the same test set.

\subsubsection{Dice}

The Dice coefficient is used to measure the similarity between the predicted value and the ground truth value. The measurement value varies between $0-1$, and 1 means that the two samples completely overlap. The specific formula is as shown in equation (3): $|\mathrm{A} \cap \mathrm{B}|$ represents the number of elements in common between the two samples, $|\mathrm{A}|$ represents the number of elements in the A sample, and B is the same. The dice global used in the experiment is the average of all test slices, and the dice per case is the average of all test cases.

$$
\text { Dice }=\frac{2|A \cap B|}{|A|+|B|}
$$

\subsubsection{Hausdorff}

Use Hausdorff95 to measure the distance between two point sets, defined as the formula (4), H(A, $\mathrm{B})$ is called the two-way Hausdorff distance, $\mathrm{h}(\mathrm{A}, \mathrm{B})$ is the one-way distance from point set A to point set $\mathrm{B}, \mathrm{H}(\mathrm{B}, \mathrm{A})$ is the one-way distance from point set $\mathrm{B}$ to point set $\mathrm{A}$.

$$
\begin{gathered}
H(A, B)=\max [h(A, B), h(B, A)] \\
h(A, B)=\max _{a \in A} \min _{b \in B}|| a-b|| \\
h(B, A)=\max _{b \in B} \min _{a \in A}|| b-a||
\end{gathered}
$$

\subsubsection{Accuracy, Recall, Precision}

The confusion matrix in Figure 5 shows the classification of whether a single CT slice contains ROI. The three indicators of accuracy, recall, and precision can be a good assessment of the correctness of the network's classification of whether a single slice contains ROI. Since this paper judges whether each slice contains ROI according to the segmentation result, a threshold $\mathrm{n}$ is set. When the sum of pixels of a single predicted ROI image is greater than $\mathrm{n}$, it is regarded as including ROI, otherwise, it does not. 


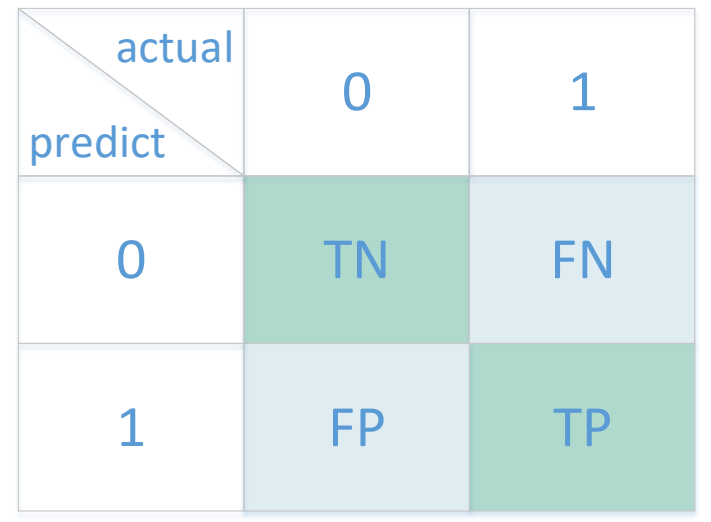

Figure 5. Confusion matrix

$$
\begin{gathered}
\text { Accuracy }=\frac{T P+T N}{T P+T N+F P+F N} \\
\text { Recall }=\frac{T P}{T P+F N} \\
\text { Precision }=\frac{T P}{T P+F P}
\end{gathered}
$$

\subsection{Experimental results and analysis}

The experiment will have two parts to prove the effectiveness of the proposed Cascaded DoubleBranch segmentation network (CDBNet). The first is the effectiveness of DBSM (Section 2.2), and the second is the effectiveness of the cascade structure.

\subsubsection{Predicted result map}

Figure 6 shows the segmentation results of the Unet network and the CDBNet network in the same test set. It can be seen that in the slice segmentation that includes ROI, CDBNet can perform better although the characteristics of ROI are different.

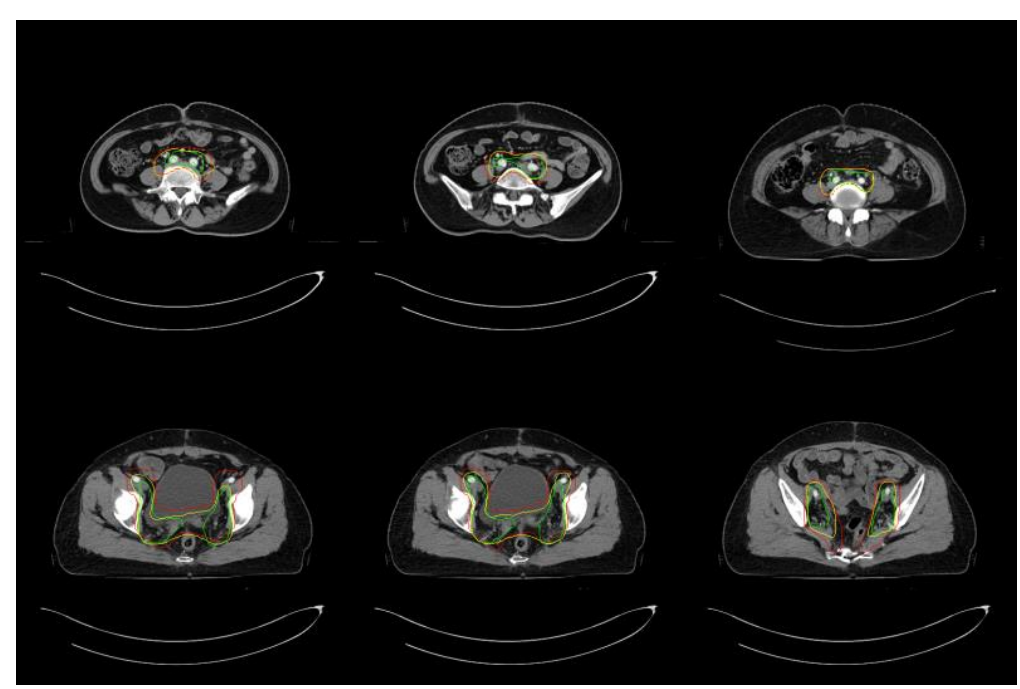

Figure 6. CTV contour result map (red is the standard contour from the physicist, yellow is the prediction result of CDBNet, and green is the prediction result of UNet) 


\subsubsection{DBSM}

DBSM is shown in Figure 4, using five-fold cross-validation in the experiment. Table 1 shows the results of the five-fold model in the same test set, where Average means the average of the five-fold results of the same network. The DBSM used in this experiment is convolved twice on the boundary branch and contains feature maps of 3 kinds of sizes. This depth is obtained by the deep ablation experiment.

Table 1 Comparison of the segmentation effect of DBSM and Unet on the cervical cancer test dataset

\begin{tabular}{|l|l|l|l|l|l|l|l|}
\hline Model & validation set & dice global & dice per case & HD & acc & recall & $\begin{array}{l}\text { dice(slices with } \\
\text { ROI) }\end{array}$ \\
\hline \multirow{4}{*}{ Unet } & 1 & & & & & & \\
\cline { 2 - 7 } & 2 & 83.26 & 83.30 & 2.67 & 92.97 & 92.94 & 61.15 \\
\cline { 2 - 8 } & 3 & 83.72 & 83.78 & 2.54 & 93.91 & 93.77 & 61.71 \\
\cline { 2 - 7 } & 4 & 84.60 & 84.63 & 2.58 & 93.13 & 91.61 & 66.35 \\
\cline { 2 - 8 } & 5 & 84.97 & 84.91 & 2.56 & 92.35 & 88.36 & 71.20 \\
\cline { 2 - 7 } & Average & 84.64 & 84.70 & 2.54 & 93.60 & 92.07 & 66.05 \\
\hline DBSM & $\mathbf{8 4 . 2 4}$ & $\mathbf{8 4 . 2 6}$ & $\mathbf{2 . 5 8}$ & $\mathbf{9 3 . 1 9}$ & $\mathbf{9 1 . 7 5}$ & 65.29 \\
\cline { 2 - 7 } & 2 & 81.70 & 81.40 & 2.80 & 88.96 & 80.07 & 73.48 \\
\cline { 2 - 7 } & 3 & 82.76 & 82.70 & 2.71 & 90.01 & 83.31 & 71.41 \\
\cline { 2 - 7 } & 4 & 84.90 & 84.94 & 2.56 & 92.97 & 92.81 & 65.76 \\
\cline { 2 - 7 } & 5 & 82.77 & 82.69 & 2.73 & 90.58 & 83.46 & 71.58 \\
\cline { 2 - 7 } & Average & 84.11 & 84.1 & 2.62 & 91.67 & 85.85 & 72.11 \\
\hline
\end{tabular}

From the average point of view, the effect of Unet network in Dice global, Dice per case, HD, acc, and recall is better than DBSM. But on the ROI slice, the Dice value of DBSM is about $5.58 \%$ higher than that of Unet. This shows that on slices with ROI, DBSM segmentation is more accurate. However, due to the poor ability of DBSM to determine whether there is an ROI in a slice, the overall Dice value is relatively low.

\subsubsection{Cascade structure}

Experiment 3.3.1 shows the excellent segmentation ability of DBSM in ROI slices, but it cannot distinguish whether the slices have ROI. So this experiment will prove that the cascade structure can integrate the segmentation ability of DBSM and the classification ability of Unet.

Table 2. Comparison of segmentation effect of cascade and non-cascade structure on cervical cancer test dataset

\begin{tabular}{|l|l|l|l|l|l|l|l|}
\hline Model & validation set & dice global & dice per case & HD & acc & recall & $\begin{array}{l}\text { dice(slices with } \\
\text { ROI) }\end{array}$ \\
\hline \multirow{4}{*}{ Unet } & 1 & 83.26 & 83.30 & 2.67 & 92.97 & 92.94 & 61.15 \\
\cline { 2 - 8 } & 2 & 83.72 & 83.78 & 2.54 & 93.91 & 93.77 & 61.71 \\
\cline { 2 - 7 } & 3 & 84.60 & 84.63 & 2.58 & 93.13 & 91.61 & 66.35 \\
\cline { 2 - 7 } & 4 & 84.97 & 84.91 & 2.56 & 92.35 & 88.36 & 71.20 \\
\cline { 2 - 7 } & 5 & 84.64 & 84.70 & 2.54 & 93.60 & 92.07 & 66.05 \\
\cline { 2 - 7 } & Average & 84.24 & 84.26 & 2.58 & 93.19 & 91.75 & 65.29 \\
\hline CDBNet & 1 & 85.86 & 85.82 & 2.51 & 92.97 & 92.94 & 68.22 \\
\cline { 2 - 7 } & 2 & 87.17 & 87.21 & 2.43 & 93.91 & 93.77 & 71.09 \\
\cline { 2 - 7 } & 3 & 86.05 & 86.08 & 2.55 & 93.13 & 91.61 & 70.17 \\
\cline { 2 - 7 } & 4 & 85.24 & 85.22 & 2.59 & 92.35 & 88.36 & 70.65 \\
\cline { 2 - 7 } & 5 & 86.38 & 86.40 & 2.49 & 93.60 & 92.07 & 70.77 \\
\cline { 2 - 7 } & Average & $\mathbf{8 6 . 1 4}$ & $\mathbf{8 6 . 1 5}$ & $\mathbf{2 . 5 1}$ & 93.19 & 91.75 & $\mathbf{7 0 . 1 8}$ \\
\hline
\end{tabular}


Unet is a non-cascaded structure, and CDBNet is a cascaded structure. It can be seen that CDBNet with a cascade structure retains both the classification ability of Unet and the segmentation ability of DBSM, so the overall Dice coefficient value can reach $86.14 \%$, which is about $1.9 \%$ higher than Unet.

\subsection{Ablation experiment}

This part conducts ablation experiments on the depth of the boundary segmentation branch module, and finds that the network performance is the best when downsampling twice. In addition, an ablation experiment was performed on the threshold $\mathrm{n}$ mentioned in 3.2.3.

\subsubsection{Experiment on the depth of the boundary branch}

This experiment randomly selected the training and verification data of the second fold in the five-fold cross-validation for network training, and showed the results of the same test set. The network is CDBNet, where CDBNet-2 means that the boundary branch is downsampled once, and there are a total of 2 types of feature maps.

Table 3. Depth comparison of boundary branches

\begin{tabular}{|l|l|l|l|l|l|l|}
\hline Model & dice global & dice per case & HD & Acc & Recall & $\begin{array}{l}\text { dice(slices } \\
\text { with ROI) }\end{array}$ \\
\hline CDBNet-2 & 86.90 & 86.93 & 2.45 & 93.91 & 93.77 & 70.34 \\
\hline CDBNet-3 & $\mathbf{8 7 . 1 7}$ & $\mathbf{8 7 . 2 1}$ & $\mathbf{2 . 4 3}$ & 93.91 & 93.77 & $\mathbf{7 1 . 0 9}$ \\
\hline CDBNet-4 & 86.93 & 86.98 & 2.45 & 93.91 & 93.77 & 70.42 \\
\hline
\end{tabular}

It can be seen from Table 3 that the structure performs best when the boundary branch is sampled twice, and Dice global can reach $87.17 \%$.

\subsubsection{Experiment on the threshold $n$}

This experiment explores the threshold $\mathrm{n}$ mentioned in 3.2.3. Use Unet to perform five-fold cross-validation training, set the value of $\mathrm{n}$ from 0 to 115, and increase by 5 each time. Figure 7 is the classification accuracy rate corresponding to different $n$, and the classification accuracy rate on the vertical axis is the average of the accuracy rates of the five-fold cross-validation in the respective validation sets. It can be seen that when the threshold is set to 65 , the overall classification effect is the best. 


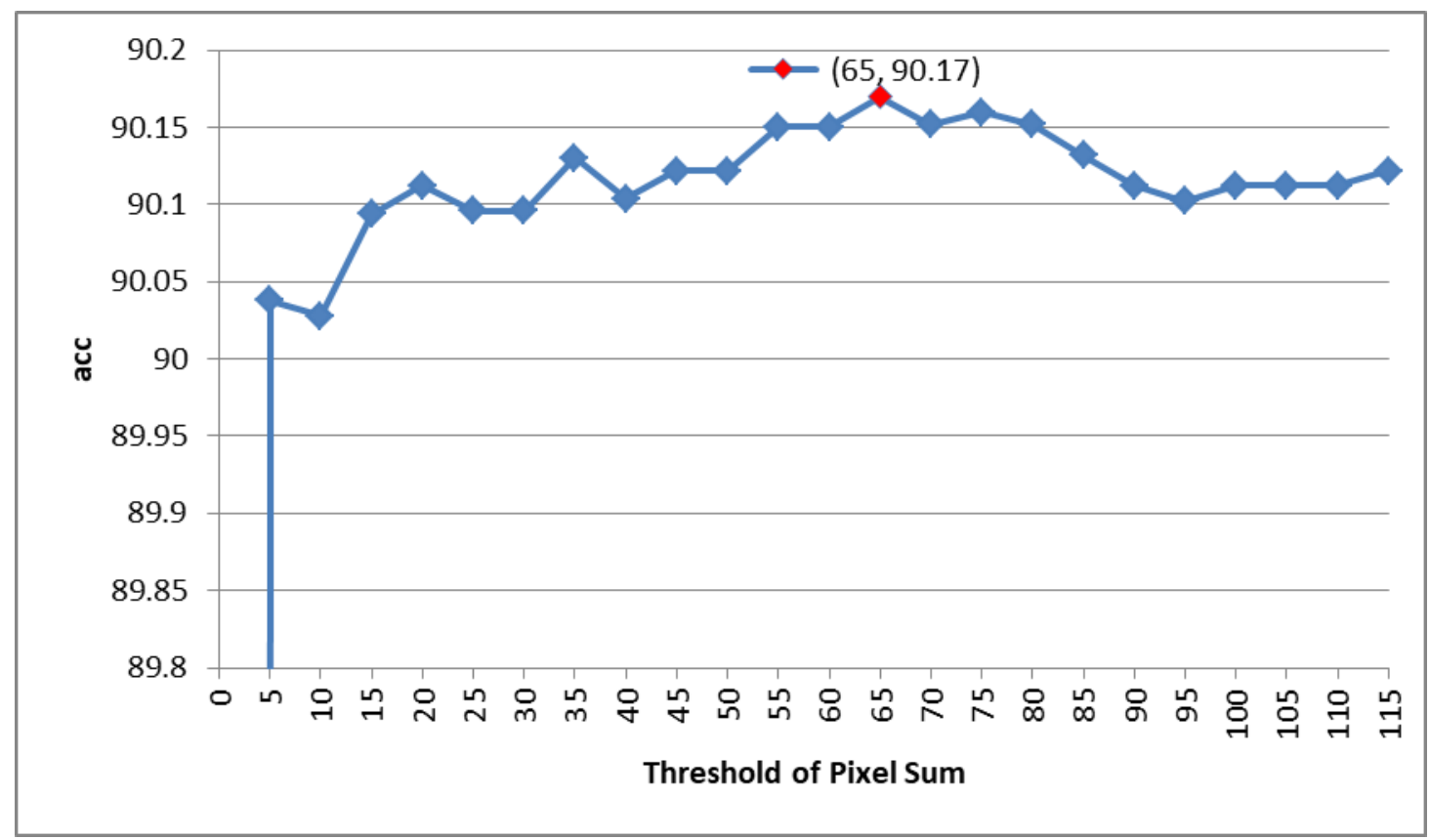

Figure 7. The relationship between the classification accuracy rate and the threshold $\mathrm{n}$

\section{CONClusion}

The outline of ROI in medical images plays a very important role in the formulation of radiotherapy treatment plans. Manual delineation is time-consuming and laborious. It usually takes an experienced physicist two or three hours to delineate all the slices of one case. This paper proposes a model CDBNet that realizes automatic ROI contour delineation on CT images, which can describe the cervical cancer risk parts in CT images. This paper mainly focuses on CTV (Clinical Target Volume). Input the CT image, the network can predict the contour and position of the CTV. The main results of this paper are as follows:

First, we use a cascade structure to solve the problem that a single model cannot take into account classification tasks and segmentation tasks at the same time. This paper designs a cascade structure formed by the classification module and the segmentation module. The classification module can predict whether each slice contains ROI. Then the classification module puts the predicted ROI slice into the segmentation module for more accurate segmentation prediction. In this way, the overall segmentation performance of a case is better, and the false-positive classification of the ROI predicted by the slice without ROI is reduced.

Secondly, we designed the CDBNet network based on the cascade structure. The DBSM module in CDBNet adds a boundary branch on the basis of Unet to supplement the boundary information so that the network has a better segmentation performance at the boundary of the ROI.

In the whole process, the research mainly explored how to improve the accuracy of CT image delineation of cases, and designed CDBNet, which has an excellent performance in both the overall classification of cases and the segmentation of ROI slices. So that the contour information during segmentation is fully preserved, the segmentation Dice value of the case can reach about $86.14 \%$, and the HD (95\%) can reach $2.51 \mathrm{~mm}$. 


\section{ACKNOWLEDGEMENTS}

This work has been supported by the scientific research projects of Quzhou Science and Technology Bureau, Zhejiang Province No.2020D012, No.2021D15, and by the major projects of the National Bureau of Statistics No.2020LD07.

\section{REFERENCES}

[1] Crane C H, Koay E J . Solutions that enable ablative radiotherapy for large liver tumors: Fractionated dose painting, simultaneous integrated protection, motion management, and computed tomography image guidance[J]. Cancer, 2016, 122(13):1974-1986.

[2] Wei D, Ahmad S, J Huo, et al. Synthesis and Inpainting-Based MR-CT Registration for ImageGuided Thermal Ablation of Liver Tumors[J]. Springer, Cham, 2019.

[3] Fujita H, Uchiyama Y, Nakagawa T, et al. Computer-aided diagnosis: The emerging of three CAD systems induced by Japanese health care needs[J]. Computer Methods \& Programs in Biomedicine, 2008, 92(3):238-248.

[4] HONG, Jae-Sung, KANEKO, et al. Automatic Liver Tumor Detection from CT[J]. IEICE transactions on information and systems, 2001, 84(6):741-748.

[5] Bilello M, Gokturk S B, Desser T, et al. Automatic detection and classification of hypodense hepatic lesions on contrast - enhanced venous - phase CT[J]. Medical Physics, 2004, 31(9).

[6] Long J, Shelhamer E, Darrell T. Fully convolutional networks for semantic segmentation[J]. IEEE Transactions on Pattern Analysis \& Machine Intelligence, 2014, 39(4):640-651.

[7] Ronneberger O, Fischer P, Brox T. U-net: Convolutional networks for biomedical image segmentation[C]//International Conference on Medical image computing and computer-assisted intervention. Springer, Cham, 2015: 234-241.

[8] Iandola F , Moskewicz M , Karayev S , et al. DenseNet: Implementing Efficient ConvNet Descriptor Pyramids[J]. Eprint Arxiv, 2014.

[9] Huang G, Liu Z, Laurens V , et al. Densely Connected Convolutional Networks[J]. IEEE Computer Society, 2016.

[10] [Zhou Z, Siddiquee M M R, Tajbakhsh N, et al. UNet++: A Nested U-Net Architecture for Medical Image Segmentation[J]. 2018.

[11] Meng L, Y Tian, Bu S . Liver tumor segmentation based on 3D convolutional neural network with double scale[J]. Journal of Applied Clinical Medical Physics, 2020, 21(1).

[12] Jose J M , Sindagi V , Hacihaliloglu I, et al. KiU-Net: Towards Accurate Segmentation of Biomedical Images using Over-complete Representations[C]// 2020.

[13] Tran S T, Cheng C H, Liu D G . A Multiple Layer U-Net, Un-Net, for Liver and Liver Tumor Segmentation in CT[J]. IEEE Access, 2020, PP(99):1-1.

[14] Wardhana G , Naghibi H , Sirmacek B , et al. Toward reliable automatic liver and tumor segmentation using convolutional neural network based on 2.5D models[J]. International Journal of Computer Assisted Radiology and Surgery, 2020, 16(12).

[15] Huang Q , Sun J , Hui D , et al. Robust liver vessel extraction using 3D U-Net with variant dice loss function[J]. Computers in Biology and Medicine, 2018, 101:S0010482518302385-.

[16] Tang Y , Tang Y , Zhu Y , et al. $E \$ \wedge 2 \$ N e t:$ An Edge Enhanced Network for Accurate Liver and Tumor Segmentation on CT Scans[J]. 2020.

[17] Chlebus G, Schenk A , Moltz J H, et al. Automatic liver tumor segmentation in CT with fully convolutional neural networks and object-based postprocessing[J]. entific Reports, 2018, 8(1).

[18] Automatic detection of contouring errors using convolutional neural networks[J]. Medical Physics, 2019, 46(11).

[19] Zhikai L, Guan H . Development And Validation Of A Deep Learning Algorithm For AutoDelineation Of Clinical Target Volume And Organs At Risk In Cervical Cancer Radiotherapy[J]. International Journal of Radiation OncologyBiologyPhysics, 2020, 108(3):e766. 


\section{AUTHORS}

Shaozhi Wu received the Ph.D. degree in Computer software and theory from the University of Electronic Science and Technology of China (UESTC), Chengdu, China,. He is currently an associate researcher at UESTC. His academic interests include artificial intelligence, deep learning applications, and pattern recognition, big data, computer network, Intelligent Sense.

Zeng $\mathbf{L i}$ is a master's student at the University of Electronic Science and Technology of China, where he studies medical image processing and region-of-interest segmentation algorithms.

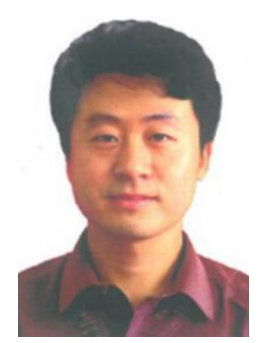

Miao Tian received the B.E. degree in electrical engineering from the University of Electronic Science and Technology of China (UESTC), Chengdu, China, in 2003 and the M.S. degree in electrical engineering from the University of Tulsa, OK, USA, in 2005 and the Ph.D. degree in electrical engineering from the University of Colorado, Boulder, USA, in 2012. From 2013 to 2014, he worked as research associate in the Earth System Science Interdisciplinary Center (ESSIC) at University of Maryland at College Park, USA. From Dec. 2014 to 2016, he worked as senior engineer at the Earth Resources Technology (ERT), Inc., Maryland. He is currently an associate researcher at UESTC. His academic interests include deep learning applications, image processing and pattern recognition, passive remote sensing, radiative transfer, instrument calibration, and antenna design.

(C) 2021 By AIRCC Publishing Corporation. This article is published under the Creative Commons Attribution (CC BY) license. 